\title{
A ZERO FREQUENCY ALTERNATIVE METHOD TO THE MOMENT METHOD OF ESTIMATION IN FINITE POISSON MIXTURES
}

\author{
DIMITRIS KARLIS and EVDOKIA XEKALAKI* \\ Department of Statistics, Athens University of Economics and Business, \\ 76, Patission St., 10434, Athens, Greece
}

(Received 6 July 2001; In final form 3 September 2002)

\begin{abstract}
The method of moments (MM) has been widely used for parametric estimation, as it is often computationally simple. Our interest focuses on the case of finite Poisson mixtures. The inefficiency of the method of moments relative to the Maximum Likelihood (ML) method is studied. Both the asymptotic efficiency as well as the small sample efficiency is examined. The case of samples that fail to lead to MM estimates is also considered. The results discourage the use of the MM estimators for two reasons; the first is that they are inefficient relative to the ML estimators and the second is the high probability of failing to lead to valid estimates. Another method, which considers replacing the third moment by the zero frequency, is examined. This method turns out to be more efficient than the moment method and not very demanding computationally.
\end{abstract}

Keywords: Moment problem; Zero frequency method; Efficiency

\section{INTRODUCTION}

The method of moments (MM) has been widely used as a simple alternative to the maximum likelihood (ML) method, mainly because of its simplicity in obtaining parameter estimates. However, it is known that the MM estimates are not as efficient as the ML estimates are and thus cautious use of them should be made. The method has also been applied to mixture models since the end of the previous century. In this paper the inappropriateness of the moment method for finite Poisson mixtures is discussed.

The probability density function (p.d.f.) of a $k$-finite mixture distribution denoted by $g_{k}(x)$ can be represented as

$$
g_{k}(x)=p_{1} f_{1}(x)+\cdots+p_{k} f_{k}(x)
$$

where $f_{i}(x), i=1, \ldots, k$ are the p.d.f.'s of the component distributions and $p_{i}$ 's are the mixing proportions. Such models are appropriate for the description of populations consisting of $k$ subpopulations in a plethora of practical situations. For example, the portfolio of an insurance company may contain several customers differing both in their predisposition to

\footnotetext{
* Corresponding author. E-mail: exek@aueb.gr
} 
accidents and in their accident risk exposure. One may thus consider that they comprise $k$ subpopulations with respect to various characteristics such as age, sex, driving or working hazards and experience. In marketing research, different categories of buyers of a product may differ in their purchasing behaviour due to factors pertaining to the appeal that the product has to them and/or their perceived utility of it as well as to its marketing. As a result, the population of buyers of a product may be heterogeneous, but may reasonably be assumed to consist of, say $k$, subpopulations which are homogeneous with respect to the aforementioned characteristics. For more examples where finite mixtures are plausible models, the interested reader is referred to Böhning (1999).

The densities $f_{i}(\cdot)$ are usually assumed to belong to the same parametric family with a parameter $\lambda$ that is allowed to vary according to a probability distribution with finite support, say $\left\{\lambda_{i}, i=1,2, \ldots, k\right\}$ termed the mixing distribution. So, the usual representation of the densities in (1) is $f_{i}(\cdot)=f\left(\cdot \mid \lambda_{i}\right), i=1,2, \ldots, k$ and the finite step distribution that assigns positive probabilities $p_{i}$ to the support points $\lambda_{i}, i=1, \ldots, k$, is considered as the mixing distribution. The number of support points $k$ corresponds to the number of components of the finite mixture. This representation assumes that the parameter $\lambda$ of the component distribution is not a constant but is itself a random variable with a distribution given by $\left\{P\left(\lambda=\lambda_{i}\right)=p_{i} ; \lambda_{i} \geq 0, i=1,2, \ldots, k\right\}$.

The MM applied to mixture models can be distinguished into two categories. The first and simplest one corresponds to the well known case where the number of components in the mixture is known, and thus a number of sample moments, is equated to the moments of the hypothesised mixture distribution. The second category has the added difficulty of unknown number of component distributions, i.e. unknown number of parameters and hence unknown number of required moments.

The method of moments was the first method employed for estimation in finite mixture problems. Pearson (1894) tried to estimate a 2-finite normal mixture by equating the population moments to the sample moments. Cohen (1967) proposed strategies for facilitating the MM for normal mixtures. Except for the case of normal mixtures described above, Rider (1961; 1962) treated several other mixtures, including the binomial, the Poisson and the exponential cases. Blischke $(1964 ; 1965)$ treated the case of binomial mixtures. John (1970) derived moment estimators and their asymptotic distributions for 2-finite mixtures of binomial, Poisson, negative binomial and hypergeometric distributions.

Tan and Chang (1972) compared the MM to the ML for a 2-finite mixture of normal distributions. Mixtures of multivariate normal distribution are treated in Day (1969) and Lindsay and Basak (1993). Tallis and Light (1968) proposed the use of fractional moments instead of integer moments, while Quandt and Ramsey (1978) proposed a method based on the moment generating function. Gupta and Huang (1981) gave a comprehensive account of such attempts up to 1980 .

Tucker (1963) considered a certain procedure to estimate the mixing distribution $G$ of a mixed Poisson distribution via the method of moments. The problem reduces to the well known moment problem (see, e.g., Shohat and Tamarkin, 1943). Brockett (1977) and Lindsay (1989), Heckman and Walker (1990) and Heckman et al. (1990) examined the case of exponential mixtures. Heckman (1990) treated the geometric mixture case, too. The main problem of such approaches remains the restricted number of components that are estimable. For example, Heckman et al. (1990), working with the exponential distribution, reported that it is very common that the moment problem has no solution for more than two moments and, hence, this restricts the applicability of the approach. Withers (1991; 1996) examined the case of moment estimators for some families of mixture distributions. Even though the existence of a solution of this moment problem is theoretically possible if the data come from the distribution under consideration, this is not always the 
case with real data. Further, in practice, using a large number of estimated moments is not advisable as the high variability of higher order moments can lead to estimates with large standard errors.

The aim of this paper is to examine further these problems. In particular, it is demonstrated that the method of moments is inferior to the maximum likelihood method of estimation for finite Poisson mixtures in terms of both small sample and asymptotic efficiency, and often fails to lead to valid parameter estimates. Moreover, whenever the ML method fails to yield parameter estimates, for finite Poisson mixtures, the MM fails too contrary to the case of normal mixtures examined in Kano (1999). The reason is that failure of the ML method to provide consistent estimates for a 2-finite Poisson mixture implies that a simple Poisson model is adequate. Then, as Lindsay and Roeder (1992) showed using the gradient function, the sample variance is less than the sample mean and hence moment estimates cannot be obtained either.

In the next section, the derivation of moment estimates for 2-finite Poisson mixtures is briefly discussed. The asymptotic efficiencies are derived in Section 3. A small sample size comparison to the ML method is also be made based on simulation. The purpose is to examine the behaviour of the MM for small sample sizes when the MM often fails to provide estimates. The case where the MM fails to lead to parameter estimates is treated in Section 4, while in Section 5 a variant of the MM is considered, which utilises the zero frequency in the place of the third moment. This leads to an increase of the efficiency of this method, especially when the mean of the sample is small whence the proportion of zeroes is large. An application of the two methods is given in Section 6 and concluding remarks are summarised in Section 7.

\section{MOMENT ESTIMATES FOR 2-FINITE POISSON MIXTURES}

In the case where the function $f$ in (1) is the probability function of the Poisson distribution, the resulting probability function defines a $k$-finite mixture of Poisson distributions, thus

$$
P(X=x)=\sum_{i=1}^{k} p_{i} \frac{\exp \left(-\lambda_{i}\right) \lambda_{i}^{x}}{x !}, \quad x=0,1,2, \ldots
$$

Here $k$ is the number of Poisson components, $\lambda_{i}>0, i=1,2, \ldots, k$, are the parameters of the Poisson distribution for each subpopulation and $p_{i}>0, i=1, \ldots, k$, are the mixing proportions with $\sum_{i=1}^{k} p_{i}=1$. For simplicity, assume that the mixing parameters $\lambda_{i}$ are in ascending order, so as to avoid identifiability problems.

Poisson mixtures are used to describe overdispersed data sets, i.e. data sets whose variance is larger than the mean. Such cases arise very often in practice and the simple Poisson distribution cannot describe them adequately. Douglas (1980) has provided a description of the properties of finite Poisson mixtures while estimation and hypothesis testing aspects have been treated by Karlis (1998).

The ML method has been applied to finite Poisson mixtures by several authors (see, for example, Titterington et al., 1985), mainly because of the easily programmed form of the EM algorithm used to derive the estimates. The moment method has also been applied by Rider (1961), John (1970) and Everitt and Hand (1981) among others. 
For the case of a 2-finite Poisson mixture the three parameters can be estimated using the first three moments of the data. The system of estimating equations is

$$
\left.\begin{array}{rl}
p_{1} \lambda_{1}+p_{2} \lambda_{2} & =\mu_{1} \\
p_{1}\left(\lambda_{1}^{2}+\lambda_{1}\right)+p_{2}\left(\lambda_{2}^{2}+\lambda_{2}\right) & =\mu_{2} \\
p_{1}\left(\lambda_{1}^{3}+3 \lambda_{1}^{2}+\lambda_{1}\right)+p_{2}\left(\lambda_{2}^{3}+3 \lambda_{2}^{2}+\lambda_{2}\right) & =\mu_{3}
\end{array}\right\}
$$

where $\mu_{k}, k=1,2,3$, are the simple sample moments. Solving this system of equations we obtain

$$
\hat{\lambda}_{1}, \hat{\lambda}_{2}=\frac{-b \pm \sqrt{D}}{2 a}
$$

where $b=\left(\mu_{3}-3 \mu_{2}+2 \mu_{1}-\mu_{1} \mu_{2}+\mu_{1}^{2}\right), a=\left(\mu_{1}^{2}-\mu_{2}+\mu_{1}\right)$ and $D=b^{2}-4 a\left(\mu_{2}^{2}-\mu_{1}^{2}+\right.$ $\left.\mu_{1} \mu_{2}-\mu_{1} \mu_{3}\right)$.

Since it is required that $\hat{\lambda}_{1}<\hat{\lambda}_{2}$, the estimate for $\lambda_{1}$ is the smallest root. This leads to

$$
\hat{p}_{2}=\frac{\mu_{1}-\hat{\lambda}_{1}}{\hat{\lambda}_{2}-\hat{\lambda}_{1}}
$$

A detailed derivation of the asymptotic variance covariance matrix of the parameter estimators is given in the Appendix.

\section{EFFICIENCY COMPARISON}

The asymptotic efficiency of the method of moments for $k=2$ is reported in Tables IA-C for several combinations of the parameters of the Poisson mixture. The entries of the tables are the values of the ratio $\left|V_{M L}\right| /\left|V_{M M}\right|$, where $|V|$ denotes the asymptotic generalised variance, obtained as the determinant of the variance-covariance matrix, and the subscripts indicate the method used. Entries lower than 1 favour the ML method. It can be seen that, for mixtures with well separated components, the efficiency is low due to the low variances of the ML methods. For components close together, the efficiency is higher, especially for mixing proportions near 0.5 .

Figure 1 depicts the asymptotic efficiency of the MM for $\lambda_{1}=1$ and various choices of the mixing proportion $p_{1}$ and the second parameter $\lambda_{2}$.

TABLE IA Asymptotic Efficiency for the Method of Moments, Relative to the Maximum Likelihood Method, for $\lambda_{1}=1$.

\begin{tabular}{lccccccccr}
\hline & \multicolumn{10}{c}{$\lambda_{2}$} \\
$p_{1}$ & 2 & 3 & 4 & 5 & 6 & 7 & 8 & 9 & 10 \\
\hline 0.1 & 0.645 & 0.322 & 0.175 & 0.112 & 0.082 & 0.065 & 0.055 & 0.049 & 0.045 \\
0.2 & 0.687 & 0.384 & 0.239 & 0.170 & 0.133 & 0.112 & 0.099 & 0.090 & 0.085 \\
0.3 & 0.729 & 0.443 & 0.297 & 0.223 & 0.181 & 0.157 & 0.142 & 0.132 & 0.126 \\
0.4 & 0.771 & 0.499 & 0.353 & 0.274 & 0.229 & 0.202 & 0.185 & 0.175 & 0.169 \\
0.5 & 0.814 & 0.556 & 0.408 & 0.325 & 0.278 & 0.249 & 0.231 & 0.221 & 0.216 \\
0.6 & 0.856 & 0.614 & 0.465 & 0.379 & 0.329 & 0.299 & 0.281 & 0.271 & 0.266 \\
0.7 & 0.898 & 0.674 & 0.526 & 0.437 & 0.384 & 0.352 & 0.334 & 0.324 & 0.320 \\
0.8 & 0.933 & 0.738 & 0.593 & 0.502 & 0.446 & 0.412 & 0.394 & 0.384 & 0.381 \\
0.9 & 0.937 & 0.796 & 0.668 & 0.579 & 0.521 & 0.485 & 0.464 & 0.454 & 0.451 \\
\hline
\end{tabular}


TABLE IB Asymptotic Efficiency for the Method of Moments, Relative to the Maximum Likelihood Method, for $\lambda_{1}={ }_{2}$.

\begin{tabular}{lccccccccr}
\hline & \multicolumn{10}{c}{$\lambda_{2}$} \\
& \multicolumn{10}{c}{$p_{1}$} & 3 & 4 & 5 & 6 & 7 & 8 & 9 & 10 & 11 \\
\hline 0.1 & 0.766 & 0.466 & 0.291 & 0.202 & 0.152 & 0.123 & 0.104 & 0.091 & 0.083 \\
0.2 & 0.813 & 0.550 & 0.385 & 0.289 & 0.232 & 0.195 & 0.172 & 0.156 & 0.145 \\
0.3 & 0.855 & 0.620 & 0.460 & 0.360 & 0.298 & 0.258 & 0.231 & 0.214 & 0.202 \\
0.4 & 0.892 & 0.682 & 0.525 & 0.423 & 0.358 & 0.315 & 0.287 & 0.268 & 0.256 \\
0.5 & 0.925 & 0.737 & 0.585 & 0.482 & 0.414 & 0.369 & 0.339 & 0.320 & 0.308 \\
0.6 & 0.952 & 0.788 & 0.641 & 0.537 & 0.467 & 0.421 & 0.390 & 0.371 & 0.359 \\
0.7 & 0.970 & 0.832 & 0.694 & 0.591 & 0.520 & 0.472 & 0.441 & 0.420 & 0.409 \\
0.8 & 0.971 & 0.864 & 0.740 & 0.643 & 0.572 & 0.524 & 0.491 & 0.471 & 0.459 \\
0.9 & 0.924 & 0.855 & 0.760 & 0.680 & 0.618 & 0.573 & 0.542 & 0.521 & 0.509 \\
\hline
\end{tabular}

TABLE IC Asymptotic Efficiency for the Method of Moments, Relative to the Maximum Likelihood Method, for $\lambda_{1}=3$.

\begin{tabular}{|c|c|c|c|c|c|c|c|c|c|}
\hline \multirow[b]{2}{*}{$p_{1}$} & \multicolumn{9}{|c|}{$\lambda_{2}$} \\
\hline & 4 & 5 & 6 & 7 & 8 & 9 & 10 & 11 & 12 \\
\hline 0.1 & 0.827 & 0.561 & 0.379 & 0.274 & 0.212 & 0.172 & 0.146 & 0.128 & 0.116 \\
\hline 0.2 & 0.870 & 0.648 & 0.484 & 0.377 & 0.308 & 0.261 & 0.229 & 0.207 & 0.192 \\
\hline 0.3 & 0.906 & 0.718 & 0.564 & 0.456 & 0.383 & 0.332 & 0.298 & 0.273 & 0.257 \\
\hline 0.4 & 0.937 & 0.776 & 0.630 & 0.522 & 0.446 & 0.394 & 0.357 & 0.332 & 0.315 \\
\hline 0.5 & 0.961 & 0.825 & 0.687 & 0.580 & 0.503 & 0.449 & 0.411 & 0.385 & 0.367 \\
\hline 0.6 & 0.978 & 0.866 & 0.737 & 0.632 & 0.555 & 0.499 & 0.460 & 0.433 & 0.415 \\
\hline 0.7 & 0.985 & 0.895 & 0.778 & 0.678 & 0.602 & 0.546 & 0.506 & 0.479 & 0.460 \\
\hline 0.8 & 0.972 & 0.906 & 0.805 & 0.715 & 0.643 & 0.588 & 0.549 & 0.522 & 0.503 \\
\hline 0.9 & 0.919 & 0.864 & 0.789 & 0.717 & 0.659 & 0.614 & 0.580 & 0.556 & 0.539 \\
\hline
\end{tabular}

In practice, the variances of the moment estimators are much higher leading to a decrease in their efficiency. The asymptotic results do not seem to hold for small to moderate sample sizes that are usually encountered in practice. For this reason, a simulation experiment was conducted in order to examine the behaviour of the moment method in the case of small

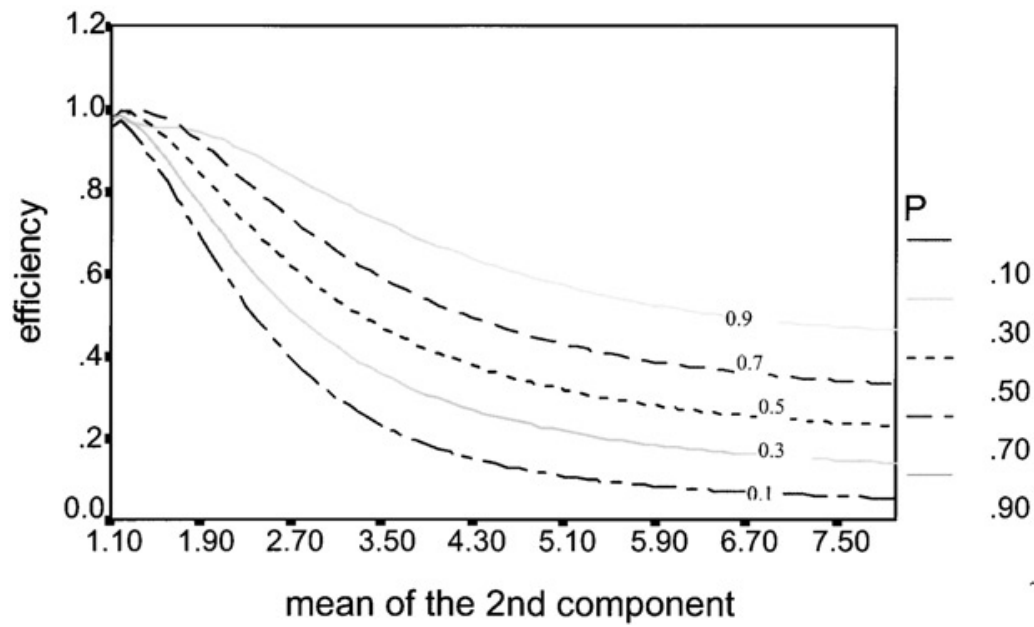

FIGURE 1 Asymptotic efficiency of the method of moments for 2-finite Poisson mixtures with $\lambda_{1}=1$. 
TABLE II Efficiency of the Moment Method Based on 1000 Simulations.

\begin{tabular}{lccccccc}
\hline & & \multicolumn{5}{c}{ Sample size } \\
\cline { 5 - 8 }$\lambda_{1}$ & $\lambda_{2}$ & $p_{1}$ & 25 & 50 & 100 & 250 & 500 \\
\hline 1 & 2 & 0.2 & 0.001 & 0.006 & 0.018 & 0.001 & 0.007 \\
1 & 2 & 0.5 & 0.001 & 0.011 & 0.019 & 0.014 & 0.113 \\
1 & 2 & 0.8 & 0.009 & 0.011 & 0.004 & 0.000 & 0.006 \\
1 & 3 & 0.2 & 0.069 & 0.080 & 0.014 & 0.532 & 0.430 \\
1 & 3 & 0.5 & 0.002 & 0.114 & 0.361 & 0.472 & 0.441 \\
1 & 3 & 0.8 & 0.030 & 0.025 & 0.127 & 0.511 & 0.473 \\
1 & 5 & 0.2 & 0.498 & 0.555 & 0.492 & 0.243 & 0.160 \\
1 & 5 & 0.5 & 0.833 & 0.524 & 0.388 & 0.371 & 0.355 \\
1 & 5 & 0.8 & 0.096 & 0.655 & 0.543 & 0.552 & 0.535 \\
1 & 8 & 0.2 & 0.469 & 0.221 & 0.161 & 0.130 & 0.107 \\
1 & 8 & 0.5 & 0.413 & 0.283 & 0.287 & 0.240 & 0.233 \\
1 & 8 & 0.8 & 0.572 & 0.557 & 0.440 & 0.446 & 0.397 \\
1 & 10 & 0.2 & 0.330 & 0.183 & 0.123 & 0.109 & 0.089 \\
1 & 10 & 0.5 & 0.346 & 0.269 & 0.223 & 0.221 & 0.210 \\
1 & 10 & 0.8 & 0.518 & 0.464 & 0.404 & 0.382 & 0.398 \\
\hline & & & & & & & \\
\hline
\end{tabular}

to moderate sample sizes. From several 2-finite Poisson mixtures 1000 samples of given sizes were simulated. Samples, which did not yield moment estimates, were dropped. This was to ensure a more concrete comparison since as is known, the variances of the ML estimates are high when the components of the mixture are close. But when the components are close, the existence of the moment method is in doubt. The generalised variance was again calculated. The results are reported in Table II.

The results are much different from those concerning asymptotic efficiency. The reason is that, for small sample sizes, there is a high probability that the moment estimates do not exist, as pointed out in the previous section. (Tables estimating this probability can be found in the next section.) Samples with a very low overdispersion usually fail to lead to moment estimates. However, applying the ML method to these samples, yields estimates with high variances (see, e.g., Hasselblad, 1969). So, samples leading to moment estimates of the parameters lead also to ML estimates of the parameters with low variances, thus making the ML method preferable to the MM.

Moreover, for many samples, especially samples of small size, the moment estimates were within the admissible range though on the boundary of admissible values, i.e. $p_{1}$ near 0 or 1 and usually a value for $\lambda_{2}$ quite large. This resulted in high variances of the moment estimates. For example, for the case $\lambda_{1}=1, \lambda_{2}=2, p_{1}=0.5$, the estimated variance of $\hat{\lambda}_{2}$ was near 85 for the moment method and 1.9 for the ML method because of a few samples that led to an estimate of $\hat{\lambda}_{2}$ whose value was near 100 . This indicates an unstable behaviour of the moment estimators. We also noted in our simulation study that the ML estimators were less biased than the MM estimators.

\section{THE EXISTENCE OF THE MOMENT ESTIMATES}

As pointed out in Section 2, samples of small or moderate size do not always lead to moment estimates. This makes the moment method not very practical because in practice the sample size is often not large enough. The estimation of the mixing distribution by the moment method is equivalent to solving a moment problem.

Shohat and Tamarkin (1943) gave necessary conditions for determining a distribution with a given finite series of moments $\left(\mu_{1}, \mu_{2}, \ldots, \mu_{s}\right)$ based on moment determinants. For a 
distribution with support in $[0,+\infty)$ (as in our case) the conditions that ensure the existence of a distribution with $s+1$ support points are

$$
\left|\begin{array}{cccc}
1 & \mu_{1} & \cdots & \mu_{s} \\
\mu_{1} & \mu_{2} & \cdots & \mu_{s+1} \\
\cdot & \cdot & \cdots & \cdot \\
\mu_{s} & \mu_{s+1} & \cdots & \mu_{2 s}
\end{array}\right| \geq 0 \text { and }\left|\begin{array}{cccc}
\mu_{1} & \mu_{2} & \cdots & \mu_{s+1} \\
\mu_{2} & \mu_{3} & \cdots & \mu_{s+2} \\
\cdot & \cdot & \cdots & \cdot \\
\mu_{s+1} & \mu_{s+2} & \cdots & \mu_{2 s+1}
\end{array}\right| \geq 0
$$

The two conditions must be satisfied simultaneously.

It can be seen that in order to estimate a mixing distribution with $k$ support points one needs the first $2 k-1$ moments. For example, for obtaining a 2-finite Poisson mixture the first three moments must satisfy the above conditions. For the first five moments the required conditions reduce to

$$
\begin{aligned}
\mu_{1} & \geq 0 \\
\mu_{2} & \geq \mu_{1}^{2} \\
\mu_{1} \mu_{3} & \geq \mu_{2}^{2} \\
\mu_{2} \mu_{4}-\mu_{1}^{2} \mu_{4}-\mu_{2}^{3}-\mu_{3}^{2}+2 \mu_{1} \mu_{2} \mu_{3} & \geq 0 \\
\mu_{1} \mu_{3} \mu_{5}-\mu_{1} \mu_{4}^{2}-\mu_{2}^{2} \mu_{5}-\mu_{3}^{2}+2 \mu_{2} \mu_{3} \mu_{4} & \geq 0
\end{aligned}
$$

The first three inequalities ensure that two support points are estimable, while all five inequalities ensure that three support points can be estimated. In order to examine how many support points one is able to estimate, a simulation experiment was carried out. From some mixed Poisson distribution, samples of varying size were generated, for a variety of parameter values. Applying the conditions given in (3), the support points that are estimable by the MM was subsequently derived. The distributions used were 2-finite Poisson mixtures, 3-finite Poisson mixtures and the negative binomial distribution with p.f.

$$
P(X=x)=\frac{\Gamma(x+b)}{x ! \Gamma(b)} p^{x}(1-p)^{b}, \quad x=0,1, \ldots ; \quad b>0, \quad 0<p<1
$$

which is a famous example of a Poisson mixture distribution.

The relationships between the moments of the mixed Poisson distribution to those of the mixing distribution are given by the following formulae

$$
\begin{aligned}
E(\lambda) & =E(X) \\
E\left(\lambda^{2}\right) & =E\left(X^{2}\right)-E(X) \\
E\left(\lambda^{3}\right) & =E\left(X^{3}\right)-3 E\left(X^{2}\right)+2 E(X) \\
E\left(\lambda^{4}\right) & =E\left(X^{4}\right)-6 E\left(X^{3}\right)+11 E\left(X^{2}\right)-6 E(X) \\
E\left(\lambda^{5}\right) & =E\left(X^{5}\right)-10 E\left(X^{4}\right)+35 E\left(X^{3}\right)-50 E\left(X^{2}\right)+24 E(X) .
\end{aligned}
$$

For the simulation experiment, the moments of the mixing distribution were estimated from the sample and substituting the theoretical moments $E\left(X^{r}\right)$ by their sample counterparts given in (5). The validity of the conditions given in (3) was subsequently checked. For each sample we found how many support points can be estimated. Tables IIIA-C summarise the results of this simulation experiment. Their entries represent the proportion of times the MM failed in estimating a solution with $k$ support points $(k=2,3)$ in 10,000 replications.

It is evident from the results that the obtained estimates have a few support points. For example, looking at Table IIIB, one can see that for small to moderate sample sizes the MM fails to give 2 support points, even though we sampled from a 2-finite distribution. 
TABLE IIIA Proportion of Times the Moment Method Failed to Give Estimates with $k$ Support Points in 10,000 Replications. Data were Generated from a Negative Binomial Distribution with Parameters $b, p$.

\begin{tabular}{|c|c|c|c|c|c|c|c|c|c|}
\hline \multirow[b]{3}{*}{$b$} & \multirow[b]{3}{*}{$p$} & \multicolumn{8}{|c|}{ Number of support points } \\
\hline & & \multicolumn{2}{|c|}{$\begin{array}{c}(\text { Sample } \\
\text { size }=50)\end{array}$} & \multicolumn{2}{|c|}{$\begin{array}{c}\text { (Sample } \\
\text { size }=100)\end{array}$} & \multicolumn{2}{|c|}{$\begin{array}{c}\text { (Sample } \\
\text { size }=250)\end{array}$} & \multicolumn{2}{|c|}{$\begin{array}{c}\text { (Sample } \\
\text { size }=500)\end{array}$} \\
\hline & & 2 & 3 & 2 & 3 & 2 & 3 & 2 & 3 \\
\hline \multirow[t]{19}{*}{0.50} & 0.05 & 0.00 & 0.03 & 0.00 & 0.00 & 0.00 & 0.00 & 0.00 & 0.00 \\
\hline & 0.10 & 0.01 & 0.33 & 0.00 & 0.07 & 0.00 & 0.00 & 0.00 & 0.00 \\
\hline & 0.15 & 0.01 & 0.69 & 0.00 & 0.31 & 0.00 & 0.05 & 0.00 & 0.00 \\
\hline & 0.20 & 0.00 & 0.92 & 0.01 & 0.59 & 0.00 & 0.19 & 0.00 & 0.04 \\
\hline & 0.25 & 0.03 & 0.98 & 0.01 & 0.82 & 0.00 & 0.41 & 0.00 & 0.16 \\
\hline & 0.30 & 0.08 & 1.00 & 0.01 & 0.94 & 0.00 & 0.61 & 0.00 & 0.34 \\
\hline & 0.35 & 0.15 & 1.00 & 0.03 & 0.99 & 0.00 & 0.79 & 0.00 & 0.54 \\
\hline & 0.40 & 0.23 & 1.00 & 0.08 & 1.00 & 0.00 & 0.91 & 0.00 & 0.72 \\
\hline & 0.45 & 0.33 & 1.00 & 0.14 & 1.00 & 0.02 & 0.98 & 0.01 & 0.87 \\
\hline & 0.50 & 0.43 & 1.00 & 0.22 & 1.00 & 0.04 & 0.99 & 0.00 & 0.96 \\
\hline & 0.55 & 0.53 & 1.00 & 0.33 & 1.00 & 0.12 & 1.00 & 0.03 & 0.99 \\
\hline & 0.60 & 0.61 & 1.00 & 0.42 & 1.00 & 0.19 & 1.00 & 0.06 & 1.00 \\
\hline & 0.65 & 0.70 & 1.00 & 0.52 & 1.00 & 0.29 & 1.00 & 0.14 & 1.00 \\
\hline & 0.70 & 0.78 & 1.00 & 0.63 & 1.00 & 0.39 & 1.00 & 0.23 & 1.00 \\
\hline & 0.75 & 0.86 & 1.00 & 0.73 & 1.00 & 0.50 & 1.00 & 0.35 & 1.00 \\
\hline & 0.80 & 0.94 & 1.00 & 0.85 & 1.00 & 0.66 & 1.00 & 0.54 & 1.00 \\
\hline & 0.85 & 0.95 & 0.98 & 0.92 & 1.00 & 0.79 & 1.00 & 0.66 & 1.00 \\
\hline & 0.90 & 0.93 & 0.93 & 1.00 & 1.00 & 1.00 & 1.00 & 1.00 & 1.00 \\
\hline & 0.95 & 0.73 & 0.73 & 0.92 & 0.92 & 1.00 & 1.00 & 1.00 & 1.00 \\
\hline \multirow[t]{19}{*}{1.00} & 0.05 & 0.00 & 0.00 & 0.00 & 0.00 & 0.00 & 0.00 & 0.00 & 0.00 \\
\hline & 0.10 & 0.00 & 0.07 & 0.00 & 0.00 & 0.00 & 0.00 & 0.00 & 0.00 \\
\hline & 0.15 & 0.00 & 0.31 & 0.00 & 0.07 & 0.00 & 0.00 & 0.00 & 0.00 \\
\hline & 0.20 & 0.00 & 0.57 & 0.00 & 0.25 & 0.00 & 0.04 & 0.00 & 0.00 \\
\hline & 0.25 & 0.00 & 0.78 & 0.01 & 0.47 & 0.00 & 0.14 & 0.00 & 0.03 \\
\hline & 0.30 & 0.00 & 0.92 & 0.00 & 0.67 & 0.00 & 0.31 & 0.00 & 0.11 \\
\hline & 0.35 & 0.04 & 0.98 & 0.00 & 0.83 & 0.00 & 0.49 & 0.01 & 0.25 \\
\hline & 0.40 & 0.08 & 1.00 & 0.01 & 0.93 & 0.00 & 0.68 & 0.00 & 0.44 \\
\hline & 0.45 & 0.16 & 1.00 & 0.04 & 0.98 & 0.00 & 0.81 & 0.00 & 0.61 \\
\hline & 0.50 & 0.24 & 1.00 & 0.09 & 1.00 & 0.01 & 0.92 & 0.01 & 0.79 \\
\hline & 0.55 & 0.33 & 1.00 & 0.15 & 1.00 & 0.02 & 0.96 & 0.00 & 0.88 \\
\hline & 0.60 & 0.42 & 1.00 & 0.24 & 1.00 & 0.07 & 0.99 & 0.01 & 0.94 \\
\hline & 0.65 & 0.52 & 1.00 & 0.34 & 1.00 & 0.14 & 1.00 & 0.04 & 0.98 \\
\hline & 0.70 & 0.63 & 1.00 & 0.44 & 1.00 & 0.23 & 1.00 & 0.10 & 1.00 \\
\hline & 0.75 & 0.70 & 1.00 & 0.55 & 1.00 & 0.35 & 1.00 & 0.20 & 1.00 \\
\hline & 0.80 & 0.80 & 1.00 & 0.66 & 1.00 & 0.48 & 1.00 & 0.33 & 1.00 \\
\hline & 0.85 & 0.90 & 1.00 & 0.80 & 1.00 & 0.59 & 1.00 & 0.46 & 1.00 \\
\hline & 0.90 & 0.96 & 0.99 & 0.92 & 1.00 & 0.81 & 1.00 & 0.67 & 1.00 \\
\hline & 0.95 & 0.92 & 0.92 & 0.99 & 0.99 & 1.00 & 1.00 & 1.00 & 1.00 \\
\hline \multirow[t]{19}{*}{3.00} & 0.05 & 0.00 & 0.00 & 0.00 & 0.00 & 0.00 & 0.00 & 0.00 & 0.00 \\
\hline & 0.10 & 0.00 & 0.00 & 0.00 & 0.00 & 0.00 & 0.00 & 0.00 & 0.00 \\
\hline & 0.15 & 0.00 & 0.03 & 0.00 & 0.00 & 0.00 & 0.00 & 0.00 & 0.00 \\
\hline & 0.20 & 0.01 & 0.14 & 0.00 & 0.02 & 0.00 & 0.00 & 0.00 & 0.00 \\
\hline & 0.25 & 0.00 & 0.31 & 0.00 & 0.10 & 0.00 & 0.00 & 0.00 & 0.00 \\
\hline & 0.30 & 0.00 & 0.49 & 0.00 & 0.23 & 0.00 & 0.04 & 0.00 & 0.00 \\
\hline & 0.35 & 0.01 & 0.65 & 0.01 & 0.40 & 0.01 & 0.12 & 0.00 & 0.02 \\
\hline & 0.40 & 0.01 & 0.79 & 0.01 & 0.56 & 0.01 & 0.25 & 0.00 & 0.09 \\
\hline & 0.45 & 0.02 & 0.89 & 0.00 & 0.69 & 0.00 & 0.40 & 0.01 & 0.21 \\
\hline & 0.50 & 0.05 & 0.95 & 0.00 & 0.81 & 0.01 & 0.55 & 0.00 & 0.35 \\
\hline & 0.55 & 0.10 & 0.98 & 0.02 & 0.90 & 0.01 & 0.68 & 0.00 & 0.50 \\
\hline & 0.60 & 0.18 & 1.00 & 0.06 & 0.96 & 0.00 & 0.80 & 0.01 & 0.65 \\
\hline & 0.65 & 0.27 & 1.00 & 0.11 & 0.98 & 0.01 & 0.90 & 0.00 & 0.81 \\
\hline & 0.70 & 0.38 & 1.00 & 0.22 & 1.00 & 0.05 & 0.95 & 0.00 & 0.88 \\
\hline & 0.75 & 0.49 & 1.00 & 0.32 & 1.00 & 0.13 & 0.99 & 0.03 & 0.96 \\
\hline & 0.80 & 0.59 & 1.00 & 0.45 & 1.00 & 0.25 & 1.00 & 0.13 & 0.99 \\
\hline & 0.85 & 0.71 & 1.00 & 0.58 & 1.00 & 0.41 & 1.00 & 0.27 & 1.00 \\
\hline & 0.90 & 0.80 & 1.00 & 0.70 & 1.00 & 0.58 & 1.00 & 0.46 & 1.00 \\
\hline & 0.95 & 0.96 & 1.00 & 0.91 & 1.00 & 0.79 & 1.00 & 0.67 & 1.00 \\
\hline
\end{tabular}


TABLE IIIA Continued.

\begin{tabular}{|c|c|c|c|c|c|c|c|c|c|}
\hline \multirow[b]{3}{*}{$b$} & \multirow[b]{3}{*}{$p$} & \multicolumn{8}{|c|}{ Number of support points } \\
\hline & & \multicolumn{2}{|c|}{$\begin{array}{c}\text { (Sample } \\
\text { size }=50)\end{array}$} & \multicolumn{2}{|c|}{$\begin{array}{c}\text { (Sample } \\
\text { size }=100)\end{array}$} & \multicolumn{2}{|c|}{$\begin{array}{c}(\text { Sample } \\
\text { size }=250)\end{array}$} & \multicolumn{2}{|c|}{$\begin{array}{c}\text { (Sample } \\
\text { size }=500)\end{array}$} \\
\hline & & 2 & 3 & 2 & 3 & 2 & 3 & 2 & 3 \\
\hline \multirow[t]{19}{*}{5.00} & 0.05 & 0.00 & 0.00 & 0.00 & 0.00 & 0.00 & 0.00 & 0.00 & 0.00 \\
\hline & 0.10 & 0.00 & 0.00 & 0.00 & 0.00 & 0.00 & 0.00 & 0.00 & 0.00 \\
\hline & 0.15 & 0.00 & 0.01 & 0.00 & 0.00 & 0.00 & 0.00 & 0.00 & 0.00 \\
\hline & 0.20 & 0.00 & 0.06 & 0.00 & 0.00 & 0.00 & 0.00 & 0.00 & 0.00 \\
\hline & 0.25 & 0.01 & 0.17 & 0.01 & 0.04 & 0.00 & 0.00 & 0.00 & 0.00 \\
\hline & 0.30 & 0.00 & 0.34 & 0.01 & 0.11 & 0.00 & 0.01 & 0.00 & 0.00 \\
\hline & 0.35 & 0.00 & 0.48 & 0.00 & 0.24 & 0.00 & 0.04 & 0.00 & 0.01 \\
\hline & 0.40 & 0.00 & 0.64 & 0.00 & 0.40 & 0.01 & 0.13 & 0.00 & 0.03 \\
\hline & 0.45 & 0.01 & 0.76 & 0.00 & 0.53 & 0.00 & 0.26 & 0.00 & 0.10 \\
\hline & 0.50 & 0.02 & 0.86 & 0.00 & 0.67 & 0.01 & 0.39 & 0.00 & 0.20 \\
\hline & 0.55 & 0.05 & 0.93 & 0.00 & 0.78 & 0.01 & 0.54 & 0.00 & 0.35 \\
\hline & 0.60 & 0.11 & 0.97 & 0.03 & 0.87 & 0.00 & 0.66 & 0.00 & 0.50 \\
\hline & 0.65 & 0.19 & 0.99 & 0.06 & 0.93 & 0.01 & 0.79 & 0.01 & 0.65 \\
\hline & 0.70 & 0.29 & 1.00 & 0.12 & 0.97 & 0.02 & 0.88 & 0.00 & 0.78 \\
\hline & 0.75 & 0.39 & 1.00 & 0.23 & 0.99 & 0.08 & 0.95 & 0.01 & 0.91 \\
\hline & 0.80 & 0.51 & 1.00 & 0.37 & 1.00 & 0.16 & 0.98 & 0.06 & 0.94 \\
\hline & 0.85 & 0.63 & 1.00 & 0.50 & 1.00 & 0.32 & 1.00 & 0.18 & 0.99 \\
\hline & 0.90 & 0.75 & 1.00 & 0.66 & 1.00 & 0.50 & 1.00 & 0.38 & 1.00 \\
\hline & 0.95 & 0.89 & 1.00 & 0.81 & 1.00 & 0.74 & 1.00 & 0.72 & 1.00 \\
\hline
\end{tabular}

TABLE IIIB Proportion of Times the Moment Method Failed to Give Estimates with $k$ Support Points in 10,000 Replications. Data were Generated from a 2-finite Poisson Mixture with Parameters $p_{1}, \lambda_{1}$ and $\lambda_{2}$.

\begin{tabular}{|c|c|c|c|c|c|c|c|c|c|c|}
\hline \multirow[b]{3}{*}{$p_{1}$} & \multirow[b]{3}{*}{$\lambda_{1}$} & \multirow[b]{3}{*}{$\lambda_{2}$} & \multicolumn{8}{|c|}{ Number of support points } \\
\hline & & & \multicolumn{2}{|c|}{$\begin{array}{c}\text { (Sample } \\
\text { size }=50)\end{array}$} & \multicolumn{2}{|c|}{$\begin{array}{c}\text { (Sample } \\
\text { size }=100)\end{array}$} & \multicolumn{2}{|c|}{$\begin{array}{c}\text { (Sample } \\
\text { size }=250)\end{array}$} & \multicolumn{2}{|c|}{$\begin{array}{c}(\text { Sample } \\
\text { size }=500)\end{array}$} \\
\hline & & & 2 & 3 & 2 & 3 & 2 & 3 & 2 & 3 \\
\hline \multirow[t]{10}{*}{0.10} & 1 & 2 & 0.71 & 1.00 & 0.64 & 1.00 & 0.56 & 0.99 & 0.52 & 0.98 \\
\hline & 1 & 3 & 0.62 & 1.00 & 0.55 & 0.99 & 0.43 & 0.96 & 0.35 & 0.92 \\
\hline & 1 & 4 & 0.55 & 0.99 & 0.48 & 0.97 & 0.36 & 0.91 & 0.28 & 0.85 \\
\hline & 1 & 5 & 0.51 & 0.97 & 0.44 & 0.94 & 0.32 & 0.85 & 0.23 & 0.78 \\
\hline & 2 & 3 & 0.69 & 1.00 & 0.63 & 0.99 & 0.57 & 0.98 & 0.53 & 0.98 \\
\hline & 2 & 4 & 0.59 & 0.99 & 0.51 & 0.98 & 0.41 & 0.95 & 0.32 & 0.91 \\
\hline & 2 & 5 & 0.52 & 0.98 & 0.44 & 0.96 & 0.31 & 0.89 & 0.20 & 0.83 \\
\hline & 3 & 4 & 0.68 & 1.00 & 0.64 & 0.99 & 0.59 & 0.98 & 0.54 & 0.97 \\
\hline & 3 & 5 & 0.59 & 0.99 & 0.53 & 0.98 & 0.43 & 0.95 & 0.35 & 0.92 \\
\hline & 4 & 5 & 0.66 & 0.99 & 0.64 & 0.99 & 0.58 & 0.97 & 0.54 & 0.97 \\
\hline \multirow[t]{10}{*}{0.30} & 1 & 2 & 0.63 & 1.00 & 0.54 & 1.00 & 0.39 & 0.98 & 0.30 & 0.96 \\
\hline & 1 & 3 & 0.45 & 1.00 & 0.31 & 0.97 & 0.15 & 0.90 & 0.07 & 0.83 \\
\hline & 1 & 4 & 0.32 & 0.97 & 0.20 & 0.91 & 0.07 & 0.82 & 0.01 & 0.76 \\
\hline & 1 & 5 & 0.26 & 0.94 & 0.15 & 0.86 & 0.03 & 0.77 & 0.01 & 0.73 \\
\hline & 2 & 3 & 0.63 & 1.00 & 0.55 & 0.99 & 0.44 & 0.98 & 0.32 & 0.95 \\
\hline & 2 & 4 & 0.43 & 0.99 & 0.29 & 0.96 & 0.14 & 0.90 & 0.04 & 0.83 \\
\hline & 2 & 5 & 0.27 & 0.97 & 0.14 & 0.90 & 0.03 & 0.82 & 0.00 & 0.76 \\
\hline & 3 & 4 & 0.63 & 0.99 & 0.56 & 0.99 & 0.46 & 0.97 & 0.38 & 0.95 \\
\hline & 3 & 5 & 0.45 & 0.99 & 0.32 & 0.96 & 0.16 & 0.90 & 0.06 & 0.85 \\
\hline & 4 & 5 & 0.62 & 0.99 & 0.57 & 0.98 & 0.48 & 0.96 & 0.40 & 0.94 \\
\hline \multirow[t]{4}{*}{0.50} & 1 & 2 & 0.58 & 1.00 & 0.47 & 1.00 & 0.28 & 0.98 & 0.16 & 0.95 \\
\hline & 1 & 3 & 0.31 & 1.00 & 0.16 & 0.97 & 0.04 & 0.89 & 0.00 & 0.82 \\
\hline & 1 & 4 & 0.16 & 0.98 & 0.04 & 0.91 & 0.00 & 0.82 & 0.01 & 0.78 \\
\hline & 1 & 5 & 0.08 & 0.94 & 0.01 & 0.85 & 0.00 & 0.77 & 0.00 & 0.74 \\
\hline
\end{tabular}


TABLE IIIB Continued.

\begin{tabular}{|c|c|c|c|c|c|c|c|c|c|c|}
\hline \multirow[b]{3}{*}{$p_{1}$} & \multirow[b]{3}{*}{$\lambda_{1}$} & \multirow[b]{3}{*}{$\lambda_{2}$} & \multicolumn{8}{|c|}{ Number of support points } \\
\hline & & & \multicolumn{2}{|c|}{$\begin{array}{c}\text { (Sample } \\
\text { size }=50)\end{array}$} & \multicolumn{2}{|c|}{$\begin{array}{c}\text { (Sample } \\
\text { size }=100)\end{array}$} & \multicolumn{2}{|c|}{$\begin{array}{c}\text { (Sample } \\
\text { size }=250)\end{array}$} & \multicolumn{2}{|c|}{$\begin{array}{c}\text { (Sample } \\
\text { size }=500)\end{array}$} \\
\hline & & & 2 & 3 & 2 & 3 & 2 & 3 & 2 & 3 \\
\hline & 2 & 3 & 0.60 & 1.00 & 0.51 & 0.99 & 0.36 & 0.98 & 0.22 & 0.94 \\
\hline & 2 & 4 & 0.31 & 0.99 & 0.16 & 0.96 & 0.05 & 0.90 & 0.00 & 0.85 \\
\hline & 2 & 5 & 0.12 & 0.97 & 0.03 & 0.91 & 0.00 & 0.82 & 0.00 & 0.76 \\
\hline & 3 & 4 & 0.61 & 1.00 & 0.54 & 0.99 & 0.41 & 0.97 & 0.30 & 0.95 \\
\hline & 3 & 5 & 0.36 & 0.99 & 0.21 & 0.96 & 0.05 & 0.89 & 0.01 & 0.85 \\
\hline & 4 & 5 & 0.60 & 0.99 & 0.54 & 0.98 & 0.44 & 0.96 & 0.34 & 0.94 \\
\hline \multirow[t]{10}{*}{0.70} & 1 & 2 & 0.58 & 1.00 & 0.45 & 1.00 & 0.27 & 0.99 & 0.13 & 0.96 \\
\hline & 1 & 3 & 0.23 & 1.00 & 0.08 & 0.98 & 0.00 & 0.92 & 0.00 & 0.87 \\
\hline & 1 & 4 & 0.05 & 0.99 & 0.00 & 0.94 & 0.01 & 0.85 & 0.00 & 0.79 \\
\hline & 1 & 5 & 0.01 & 0.97 & 0.00 & 0.89 & 0.00 & 0.81 & 0.00 & 0.76 \\
\hline & 2 & 3 & 0.61 & 1.00 & 0.51 & 0.99 & 0.37 & 0.98 & 0.24 & 0.96 \\
\hline & 2 & 4 & 0.30 & 1.00 & 0.13 & 0.97 & 0.02 & 0.92 & 0.01 & 0.87 \\
\hline & 2 & 5 & 0.08 & 0.98 & 0.01 & 0.93 & 0.00 & 0.85 & 0.00 & 0.80 \\
\hline & 3 & 4 & 0.61 & 1.00 & 0.54 & 0.99 & 0.42 & 0.97 & 0.32 & 0.95 \\
\hline & 3 & 5 & 0.35 & 0.99 & 0.21 & 0.97 & 0.05 & 0.91 & 0.01 & 0.87 \\
\hline & 4 & 5 & 0.61 & 0.99 & 0.56 & 0.98 & 0.45 & 0.97 & 0.38 & 0.95 \\
\hline \multirow[t]{10}{*}{0.90} & 1 & 2 & 0.67 & 1.00 & 0.59 & 1.00 & 0.44 & 1.00 & 0.29 & 0.99 \\
\hline & 1 & 3 & 0.39 & 1.00 & 0.22 & 1.00 & 0.05 & 0.99 & 0.00 & 0.96 \\
\hline & 1 & 4 & 0.18 & 1.00 & 0.05 & 1.00 & 0.00 & 0.95 & 0.01 & 0.90 \\
\hline & 1 & 5 & 0.06 & 1.00 & 0.01 & 0.98 & 0.00 & 0.91 & 0.01 & 0.84 \\
\hline & 2 & 3 & 0.68 & 1.00 & 0.61 & 1.00 & 0.51 & 0.99 & 0.43 & 0.98 \\
\hline & 2 & 4 & 0.49 & 1.00 & 0.34 & 0.99 & 0.14 & 0.97 & 0.04 & 0.94 \\
\hline & 2 & 5 & 0.27 & 1.00 & 0.11 & 0.99 & 0.01 & 0.94 & 0.01 & 0.90 \\
\hline & 3 & 4 & 0.68 & 1.00 & 0.62 & 0.99 & 0.56 & 0.98 & 0.49 & 0.97 \\
\hline & 3 & 5 & 0.52 & 1.00 & 0.42 & 0.99 & 0.23 & 0.96 & 0.11 & 0.93 \\
\hline & 4 & 5 & 0.68 & 1.00 & 0.63 & 0.99 & 0.57 & 0.98 & 0.52 & 0.97 \\
\hline
\end{tabular}

TABLE IIIC Proportion of Times the Moment Method Failed to Give Estimates with $k$ Support Points in 10,000 Replications. Data were Generated from a 3-finite Poisson Mixture with Parameters $p_{1}, p_{2}, \lambda_{1}, \lambda_{2}$ and $\lambda_{3}$.

\begin{tabular}{|c|c|c|c|c|c|c|c|c|c|c|c|c|}
\hline \multirow[b]{3}{*}{$p_{1}$} & \multirow[b]{3}{*}{$p_{2}$} & \multirow[b]{3}{*}{$\lambda_{1}$} & \multirow[b]{3}{*}{$\lambda_{2}$} & \multirow[b]{3}{*}{$\lambda_{3}$} & \multicolumn{8}{|c|}{ Number of support points } \\
\hline & & & & & \multicolumn{2}{|c|}{$\begin{array}{c}\text { (Sample } \\
\text { size }=50)\end{array}$} & \multicolumn{2}{|c|}{$\begin{array}{c}\text { (Sample } \\
\text { size }=100)\end{array}$} & \multicolumn{2}{|c|}{$\begin{array}{c}(\text { Sample } \\
\text { size }=250)\end{array}$} & \multicolumn{2}{|c|}{$\begin{array}{c}(\text { Sample } \\
\text { size }=500)\end{array}$} \\
\hline & & & & & 2 & 3 & 2 & 3 & 2 & 3 & 2 & 3 \\
\hline \multirow[t]{12}{*}{0.25} & \multirow[t]{6}{*}{0.2} & \multirow[t]{2}{*}{1} & 2 & 6 & 0.07 & 0.90 & 0.02 & 0.82 & 0.00 & 0.73 & 0.01 & 0.69 \\
\hline & & & 4 & 6 & 0.17 & 0.90 & 0.08 & 0.82 & 0.00 & 0.70 & 0.00 & 0.63 \\
\hline & & \multirow[t]{2}{*}{2} & 2 & 6 & 0.05 & 0.91 & 0.01 & 0.85 & 0.00 & 0.77 & 0.00 & 0.72 \\
\hline & & & 4 & 6 & 0.16 & 0.94 & 0.06 & 0.86 & 0.00 & 0.75 & 0.01 & 0.69 \\
\hline & & \multirow[t]{2}{*}{3} & 2 & 6 & 0.10 & 0.93 & 0.02 & 0.87 & 0.00 & 0.77 & 0.00 & 0.72 \\
\hline & & & 4 & 6 & 0.25 & 0.97 & 0.11 & 0.91 & 0.01 & 0.82 & 0.01 & 0.76 \\
\hline & \multirow[t]{6}{*}{0.4} & \multirow[t]{2}{*}{1} & 2 & 4 & 0.18 & 0.99 & 0.07 & 0.95 & 0.01 & 0.85 & 0.00 & 0.78 \\
\hline & & & 6 & 4 & 0.15 & 0.92 & 0.05 & 0.82 & 0.01 & 0.69 & 0.00 & 0.59 \\
\hline & & \multirow[t]{2}{*}{2} & 2 & 4 & 0.28 & 0.99 & 0.14 & 0.97 & 0.03 & 0.91 & 0.00 & 0.86 \\
\hline & & & 6 & 4 & 0.14 & 0.95 & 0.04 & 0.87 & 0.00 & 0.76 & 0.00 & 0.67 \\
\hline & & \multirow[t]{2}{*}{3} & 2 & 4 & 0.39 & 0.99 & 0.23 & 0.97 & 0.08 & 0.92 & 0.01 & 0.85 \\
\hline & & & 6 & 4 & 0.23 & 0.97 & 0.11 & 0.93 & 0.02 & 0.85 & 0.01 & 0.79 \\
\hline \multirow[t]{6}{*}{0.50} & \multirow[t]{6}{*}{0.2} & \multirow[t]{2}{*}{1} & 4 & 2 & 0.14 & 1.00 & 0.04 & 0.97 & 0.00 & 0.87 & 0.01 & 0.79 \\
\hline & & & 6 & 2 & 0.00 & 0.97 & 0.00 & 0.89 & 0.00 & 0.78 & 0.00 & 0.71 \\
\hline & & \multirow[t]{2}{*}{2} & 4 & 2 & 0.35 & 1.00 & 0.20 & 0.99 & 0.04 & 0.94 & 0.00 & 0.89 \\
\hline & & & 6 & 2 & 0.02 & 0.98 & 0.00 & 0.93 & 0.00 & 0.85 & 0.01 & 0.80 \\
\hline & & \multirow[t]{2}{*}{3} & 4 & 2 & 0.50 & 1.00 & 0.35 & 0.98 & 0.18 & 0.94 & 0.08 & 0.89 \\
\hline & & & 6 & 2 & 0.08 & 0.98 & 0.02 & 0.93 & 0.01 & 0.83 & 0.00 & 0.75 \\
\hline
\end{tabular}


TABLE IIIC Continued.

\begin{tabular}{|c|c|c|c|c|c|c|c|c|c|c|c|c|}
\hline \multirow[b]{3}{*}{$p_{1}$} & \multirow[b]{3}{*}{$p_{2}$} & \multirow[b]{3}{*}{$\lambda_{1}$} & \multirow[b]{3}{*}{$\lambda_{2}$} & \multirow[b]{3}{*}{$\lambda_{3}$} & \multicolumn{8}{|c|}{ Number of support points } \\
\hline & & & & & \multicolumn{2}{|c|}{$\begin{array}{c}\text { (Sample } \\
\text { size }=50)\end{array}$} & \multicolumn{2}{|c|}{$\begin{array}{c}\text { (Sample } \\
\text { size }=100)\end{array}$} & \multicolumn{2}{|c|}{$\begin{array}{c}(\text { Sample } \\
\text { size }=250)\end{array}$} & \multicolumn{2}{|c|}{$\begin{array}{c}\text { (Sample } \\
\text { size }=500)\end{array}$} \\
\hline & & & & & 2 & 3 & 2 & 3 & 2 & 3 & 2 & 3 \\
\hline & \multirow[t]{6}{*}{0.4} & \multirow[t]{2}{*}{1} & 2 & 6 & 0.04 & 1.00 & 0.00 & 0.95 & 0.01 & 0.84 & 0.00 & 0.75 \\
\hline & & & 4 & 6 & 0.06 & 0.94 & 0.00 & 0.83 & 0.00 & 0.68 & 0.00 & 0.56 \\
\hline & & \multirow[t]{2}{*}{2} & 2 & 6 & 0.13 & 1.00 & 0.03 & 0.98 & 0.00 & 0.92 & 0.00 & 0.87 \\
\hline & & & 4 & 6 & 0.13 & 0.98 & 0.03 & 0.92 & 0.00 & 0.79 & 0.00 & 0.69 \\
\hline & & \multirow[t]{2}{*}{3} & 2 & 6 & 0.19 & 0.99 & 0.07 & 0.96 & 0.00 & 0.87 & 0.00 & 0.79 \\
\hline & & & 4 & 6 & 0.38 & 0.99 & 0.24 & 0.97 & 0.06 & 0.91 & 0.01 & 0.85 \\
\hline
\end{tabular}

The same is true in Table IIIC for 3-finite Poisson mixtures. Even for large sample sizes, the method fails to reconstruct the true mixing distribution with 3 support points. For the negative binomial distribution, it can be seen from Table IIIA, that as the overdispersion decreases, the method of moments fail. For this particular parameterisation of the negative binomial distribution, the index of dispersion equals $1+b((1-p) / p)$ and decreases as the value of $p$ increases. It is interesting to note that when the mixing distribution of $\lambda$ is a continuous distribution (as in the case of the negative binomial distribution), it is possible to estimate only a few support points using the MM. This is true even when the sample size is relatively large. Note also that the moment method often fails to lead to estimates of the parameters of the negative binomial distribution (see Johnson et al., 1992).

Similar results were obtained by Heckman et al. (1990) for the case of finite exponential mixtures. They also reported that very few support points ( 2 or 3 ) are estimable through the MM.

In concluding, one may say that the moment method has some disadvantages, which lessen its practical value: Parameter estimates are not always obtainable and, when they are, their variances are much higher than those of the corresponding ML estimates.

\section{THE ZERO FREQUENCY METHOD}

Because of the high sampling variance of the third moment, one may look for some other function to replace it. A usual choice for discrete distributions is the zero relative frequency of the data set which is equated to the probability of zero under the assumed distribution (see, e.g., Kemp and Kemp, 1988). So, the resulting system of equations is

$$
\left.\begin{array}{r}
p_{1} \lambda_{1}+p_{2} \lambda_{2}=\mu_{1} \\
p_{1}\left(\lambda_{1}^{2}+\lambda_{1}\right)+p_{2}\left(\lambda_{2}^{2}+\lambda_{2}\right)=\mu_{2} \\
p_{1} \exp \left(-\lambda_{1}\right)+p_{2} \exp \left(-\lambda_{2}\right)=P_{0}
\end{array}\right\},
$$

where $P_{0}$ is the observed proportion of zero values in the sample. For distributions with a high probability at 0 , this method is expected to work satisfactorily. Moreover, due to the lower variance of the proportion of zeroes relative to that of the third sample moment, the method is expected to have a higher efficiency.

Expressing $p_{1}$ and $\lambda_{2}$ in the third equation in terms of $\lambda_{1}$, we obtain

$$
(1-a) \exp \left(-\lambda_{1}\right)+a \exp (-b)=P_{0}
$$

where $b=\left(\left(\mu_{2}-\mu_{1}-\mu_{1} \lambda_{1}\right) /\left(\mu_{1}-\lambda_{1}\right)\right)$ and $a=\left(\left(\mu_{1}-\lambda_{1}\right) /\left(b-\lambda_{1}\right)\right)$. 
Equation (6) is non-linear and a numerical technique is required for solving it. A simple iterative scheme such as the Newton-Raphson method can be utilised. A 'good' initial choice for the value of $\lambda_{1}$ would be to use $\lambda_{1}^{(0)}=\ln P_{0}$, i.e. the value that $\lambda_{1}$ would have if a simple Poisson model were assumed. The estimate is then updated using

$$
\lambda_{1}^{(i+1)}=\lambda_{1}^{(i)}-\frac{f\left(\lambda_{1}^{(i)}\right)}{f^{\prime}\left(\lambda_{1}^{(i)}\right)},
$$

where $f\left(\lambda_{1}\right)=(1-a) \exp \left(-\lambda_{1}\right)+a \exp (-b)-P_{0}$,

$$
f^{\prime}\left(\lambda_{1}\right)=-a^{\prime} \exp \left(-\lambda_{1}\right)-(1-a) \exp \left(-\lambda_{1}\right)+a^{\prime} \exp (-b)-b^{\prime} \exp (-b) a .
$$

\begin{tabular}{|c|c|c|c|c|c|c|c|c|c|}
\hline \multirow[b]{2}{*}{$p_{1}$} & \multicolumn{9}{|c|}{$\lambda_{2}$} \\
\hline & 2 & 3 & 4 & 5 & 6 & 7 & 8 & 9 & 10 \\
\hline 0.1 & 0.949 & 0.879 & 0.762 & 0.661 & 0.585 & 0.528 & 0.486 & 0.458 & 0.439 \\
\hline 0.2 & 0.935 & 0.878 & 0.778 & 0.687 & 0.611 & 0.553 & 0.511 & 0.481 & 0.460 \\
\hline 0.3 & 0.917 & 0.868 & 0.773 & 0.682 & 0.606 & 0.549 & 0.508 & 0.479 & 0.460 \\
\hline 0.4 & 0.893 & 0.850 & 0.757 & 0.666 & 0.592 & 0.537 & 0.498 & 0.471 & 0.454 \\
\hline 0.5 & 0.861 & 0.821 & 0.730 & 0.642 & 0.572 & 0.520 & 0.484 & 0.460 & 0.445 \\
\hline 0.6 & 0.819 & 0.782 & 0.695 & 0.612 & 0.547 & 0.500 & 0.467 & 0.446 & 0.433 \\
\hline 0.7 & 0.761 & 0.727 & 0.647 & 0.572 & 0.514 & 0.472 & 0.445 & 0.427 & 0.416 \\
\hline 0.8 & 0.677 & 0.645 & 0.576 & 0.513 & 0.465 & 0.432 & 0.410 & 0.396 & 0.389 \\
\hline 0.9 & 0.537 & 0.505 & 0.451 & 0.405 & 0.372 & 0.350 & 0.337 & 0.330 & 0.328 \\
\hline
\end{tabular}

TABLE IVA Asymptotic Efficiencies for the Method of Zero Frequency Relative to the ML Method, for $\lambda_{1}=1$.

TABLE IVB Asymptotic Efficiencies for the Method of Zero Frequency Relative to the ML Method, for $\lambda_{1}=2$.

\begin{tabular}{cccccccccc}
\hline & \multicolumn{10}{c}{$\lambda_{2}$} \\
\cline { 2 - 9 }$p_{1}$ & 3 & 4 & 5 & 6 & 7 & 8 & 9 & 10 & 11 \\
\hline 0.1 & 0.753 & 0.764 & 0.680 & 0.571 & 0.474 & 0.400 & 0.346 & 0.308 & 0.281 \\
0.2 & 0.712 & 0.708 & 0.621 & 0.520 & 0.435 & 0.372 & 0.326 & 0.294 & 0.271 \\
0.3 & 0.669 & 0.653 & 0.567 & 0.474 & 0.399 & 0.344 & 0.305 & 0.278 & 0.258 \\
0.4 & 0.624 & 0.598 & 0.516 & 0.432 & 0.367 & 0.319 & 0.285 & 0.262 & 0.246 \\
0.5 & 0.575 & 0.542 & 0.466 & 0.392 & 0.335 & 0.294 & 0.265 & 0.245 & 0.232 \\
0.6 & 0.521 & 0.483 & 0.414 & 0.350 & 0.302 & 0.267 & 0.243 & 0.227 & 0.216 \\
0.7 & 0.461 & 0.418 & 0.357 & 0.304 & 0.264 & 0.236 & 0.217 & 0.205 & 0.196 \\
0.8 & 0.389 & 0.342 & 0.290 & 0.248 & 0.217 & 0.196 & 0.182 & 0.173 & 0.168 \\
0.9 & 0.295 & 0.240 & 0.201 & 0.171 & 0.150 & 0.136 & 0.127 & 0.122 & 0.120 \\
\hline
\end{tabular}

\begin{tabular}{|c|c|c|c|c|c|c|c|c|c|}
\hline \multirow[b]{2}{*}{$p_{1}$} & \multicolumn{9}{|c|}{$\lambda_{2}$} \\
\hline & 4 & 5 & 6 & 7 & 8 & & 10 & 11 & 12 \\
\hline 0.1 & 0.525 & 0.546 & 0.482 & 0.391 & 0.311 & 0.252 & 0.210 & 0.181 & 0.160 \\
\hline 0.2 & 0.484 & 0.479 & 0.410 & 0.330 & 0.266 & 0.219 & 0.187 & 0.164 & 0.148 \\
\hline 0.3 & 0.444 & 0.424 & 0.356 & 0.287 & 0.234 & 0.195 & 0.168 & 0.150 & 0.136 \\
\hline 0.4 & 0.405 & 0.374 & 0.312 & 0.252 & 0.207 & 0.175 & 0.153 & 0.137 & 0.126 \\
\hline 0.5 & 0.366 & 0.329 & 0.272 & 0.221 & 0.183 & 0.156 & 0.138 & 0.125 & 0.116 \\
\hline 0.6 & 0.326 & 0.284 & 0.233 & 0.191 & 0.159 & 0.137 & 0.122 & 0.112 & 0.105 \\
\hline 0.7 & 0.284 & 0.238 & 0.194 & 0.159 & 0.134 & 0.117 & 0.105 & 0.097 & 0.091 \\
\hline 0.8 & 0.238 & 0.188 & 0.152 & 0.124 & 0.105 & 0.092 & 0.083 & 0.077 & 0.074 \\
\hline 0.9 & 0.183 & 0.127 & 0.100 & 0.081 & 0.068 & 0.060 & 0.054 & 0.050 & 0.048 \\
\hline
\end{tabular}

TABLE IVC Asymptotic Efficiencies for the Method of Zero Frequency Relative to the ML Method, for $\lambda_{1}=3$. 
with $a^{\prime}=2\left(\mu_{1}^{2}-\mu_{2}+\mu_{1}\right)\left(\mu_{1}-\lambda_{1}\right) /\left(-\mu_{2}+\mu_{1}+2 \mu_{1} \lambda_{1}-\lambda_{1}^{2}\right)^{2} \quad$ and $\quad b^{\prime}=\mu_{2}-\mu_{1}-\mu_{1}^{2} /$ $\left(\mu_{1}-\lambda_{1}\right)^{2}$.

Having obtained $\hat{\lambda}_{1}$, the remaining parameters are estimated by

$$
\hat{\lambda}_{2}=\frac{\mu_{2}-\mu_{1}-\mu_{1} \hat{\lambda}_{1}}{\mu_{1}-\hat{\lambda}_{1}} \quad \text { and } \quad \hat{p}_{2}=\frac{\mu_{1}-\hat{\lambda}_{1}}{\hat{\lambda}_{2}-\hat{\lambda}_{1}} .
$$

The derivation of the asymptotic variance-covariance matrix of the parameter estimators can be found in the Appendix. The ZF estimates do not exist when the sample variance is greater than the sample mean, since in this case the first two moment equations cannot be satisfied.

Tables IVA-C present the asymptotic efficiency of the method of zero frequency. It can be seen that for distributions with a high probability at 0 , the efficiency is high. Comparing this method to the ordinary MM, one can see that it is more efficient for distributions with a low mean. This makes the zero frequency method an interesting alternative to the ML method when there are many counts at 0 . Note also that the method succeeds in providing estimates which give an excellent fit for the zero frequency. Note that as Harris (1991) pointed out, the estimated frequency of zero from the ML estimates is always greater than the observed frequency.

Figure 2 depicts the asymptotic efficiency of the zero frequency method for $\lambda_{1}=1$ and various choices of values for the mixing proportion and the second parameter. The efficiency is high for components close to the positive axis, since such 2-finite Poisson distributions have a high proportion of zeroes. For cases with a low zero frequency, the method is not satisfactory.

Figure 3 depicts the efficiency of the MM relative to that of the ZF method. The relative efficiency was calculated as the ratio of the generalised variance of the moment method divided by that of the zero frequency method. Values lower than 1 favour the MM. When the mixing proportion is high, i.e. when the zero frequency is high, the zero frequency method is superior to the moment method. Perhaps, these two methods can be used complementarily to fully exploit their behavioural features. Note that the ML method is preferable in all cases, apart from situations where the estimation of the zero frequency requires special attention.

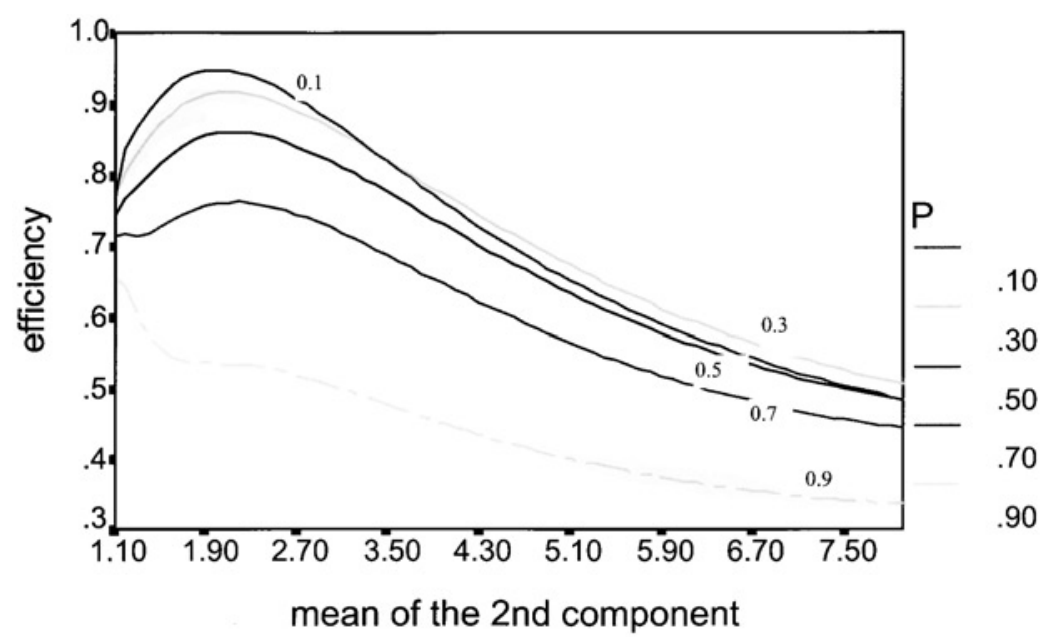

FIGURE 2 Asymptotic efficiency of the method of zero frequency for 2 -finite Poisson mixtures with $\lambda_{1}=1$. 


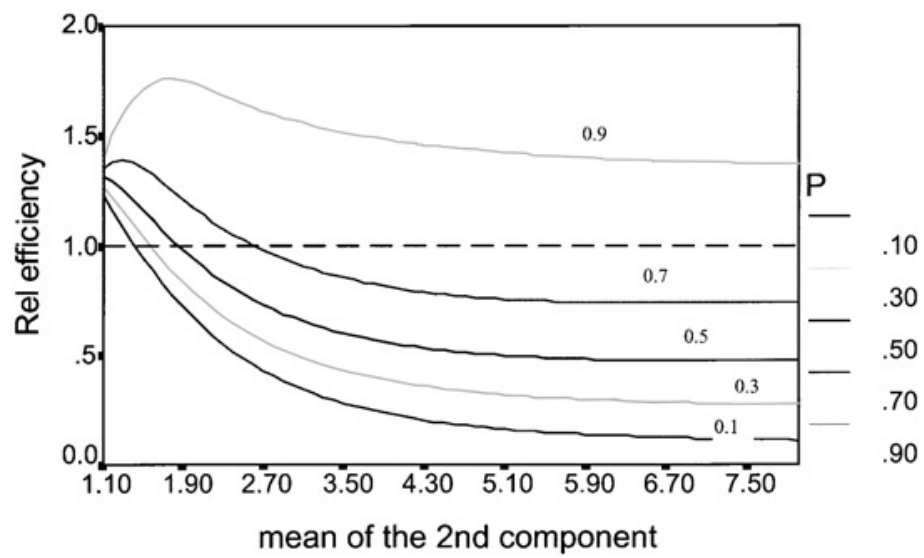

FIGURE 3 Relative efficiency of the moment and the zero frequency methods.

\section{AN APPLICATION}

Table V contains several datasets related to psychometry and accident theory. Due to the presence of overdispersion in all of the datasets, simple Poisson models would be inappropriate for describing the mechanisms that led to the data. Finite Poisson mixtures seem to constitute more plausible alternatives. So, in the sequel, finite Poisson mixtures are applied to the data. As the emphasis is on illustrating the performance of the estimation techniques discussed in the previous section and not on demonstrating the improvement of fit on the data, finite Poisson mixtures of two components only have been considered being easier to handle computationally.

As can be seen, the zero frequency of all datasets is very high. Hence, the zero frequency method is expected to lead to better estimates than the MM. The zero frequency method, the MM and the ML method were applied to these data. The results are summarized in Table VI. The jack-knife estimates of the standard errors of the parameter estimates are reported in columns 1-3. Jack-knife estimation of standard errors of the parameter estimates was considered

TABLE V Sets of Overdispersed Count Data.

\begin{tabular}{|c|c|c|c|}
\hline \multirow[b]{2}{*}{ Count variable } & \multicolumn{3}{|c|}{ Observed frequency } \\
\hline & $\begin{array}{c}\text { Absences of } \\
\text { industrial } \\
\text { workers (Sichel, 1951) }\end{array}$ & $\begin{array}{c}\text { Injury accidents } \\
\text { incurred by industrial } \\
\text { workers (Sichel, 1951) }\end{array}$ & $\begin{array}{c}\text { Injury accidents } \\
\text { incurred by bus } \\
\text { drivers (Panaretos, 1981) }\end{array}$ \\
\hline 0 & 217 & 77 & 286 \\
\hline 1 & 44 & 36 & 216 \\
\hline 2 & 29 & 24 & 92 \\
\hline 3 & 11 & 13 & 30 \\
\hline 4 & 11 & 4 & 14 \\
\hline 5 & 2 & 3 & 1 \\
\hline 6 & 4 & 2 & - \\
\hline 7 & - & 1 & - \\
\hline 8 & - & 2 & - \\
\hline 9 & - & 2 & - \\
\hline$>9$ & - & $1 *$ & - \\
\hline Mean & 0.669 & 1.345 & 0.862 \\
\hline Variance & 1.529 & 4.395 & 0.982 \\
\hline Variance/mean & 2.283 & 3.267 & 1.139 \\
\hline
\end{tabular}

*The actual count was 15 . 
TABLE VI The Estimates of the Parameters of a 2-finite Poisson Mixture for the Data of Table V.

\begin{tabular}{|c|c|c|c|c|c|c|c|c|c|}
\hline \multirow[b]{2}{*}{ Data set } & \multirow[b]{2}{*}{ Method } & \multicolumn{3}{|c|}{ Estimates } & \multicolumn{3}{|c|}{ Standard errors } & \multicolumn{2}{|c|}{$\begin{array}{l}p \text {-value of the } \\
\chi^{2} \text { goodness } \\
\text { of fit test }\end{array}$} \\
\hline & & $p_{1}$ & $\lambda_{1}$ & $\lambda_{2}$ & $p_{1}$ & $\lambda_{1}$ & $\lambda_{2}$ & $\begin{array}{l}\text { 2-finite } \\
\text { Poisson } \\
\text { mixture }\end{array}$ & $\begin{array}{c}\text { Simple } \\
\text { Poisson }\end{array}$ \\
\hline \multirow{3}{*}{$\begin{array}{l}\text { Absences } \\
\text { (Sichel, } \\
\text { 1951) }\end{array}$} & ML & 0.72721 & 0.11196 & 2.15706 & 0.05520 & 0.05359 & 0.31120 & 0.20 & \multirow{3}{*}{$<0.0001$} \\
\hline & MOM & 0.74516 & 0.12766 & 2.25523 & 0.05569 & 0.05718 & 0.13063 & 0.17 & \\
\hline & ZERO & 0.74587 & 0.12867 & 2.25818 & 0.05291 & 0.05191 & 0.13062 & 0.17 & \\
\hline \multirow{3}{*}{$\begin{array}{l}\text { Industrial accidents } \\
\text { (Sichel, } \\
\text { 1951) }\end{array}$} & ML & 0.89210 & 0.80028 & 5.86084 & 0.07710 & 0.19009 & 2.10590 & 0.04 & \multirow{3}{*}{$<0.0001$} \\
\hline & MOM & 0.92226 & 0.83891 & 7.37089 & 0.07879 & 0.12592 & 0.46833 & 0.02 & \\
\hline & ZERO & 0.84351 & 0.59350 & 5.40181 & 0.07075 & 0.09263 & 0.33977 & 0.01 & \\
\hline \multirow{3}{*}{$\begin{array}{l}\text { Road accidents } \\
\text { (Panaretos, } \\
\text { 1981) }\end{array}$} & ML & 0.52008 & 0.51898 & 1.23434 & 0.21075 & 0.15779 & 0.19484 & 0.58 & \multirow{3}{*}{0.02} \\
\hline & MOM & 0.23957 & 0.24388 & 1.05698 & 0.09751 & 0.13071 & 0.07586 & 0.29 & \\
\hline & ZERO & 0.52343 & 0.53109 & 1.22611 & 0.14159 & 0.11658 & 0.12144 & 0.55 & \\
\hline
\end{tabular}

to avoid using asymptotic standard errors with samples of small or moderate size. Alternatively, bootstrap estimation could have been considered.

For the majority of cases, the zero frequency estimates have standard errors which are smaller than those of the moment estimates, while at the same time they are smaller than those of the ML estimates for $p_{1}$ and $\lambda_{1}$. It is interesting to note that the selected data sets have a variety of shapes (long tails, excess of zeros etc) and the zero frequency method remains a competitive alternative to the ML and the moment methods.

Moreover, since it makes use of the bulk of probability mass which is concentrated towards 0 , it is less sensitive to the presence of an extreme value in the data set, as in the case of absenteeism data or to the skewness of the observed distribution as in the case of the road accident data. As revealed by the resulting estimates of the parameters, none of the remaining two methods appears to have captured the presence of the outlier in the former data set, while only the ML method appears to have coped with the short-tailedness of the latter data set comparably to the zero-frequency method.

From the practical point of view, one may be interested in the proportion of zero values, which provides information concerning the examined situation (e.g. accident free drivers). The zerofrequency method is thus appealing as it better describes this part of the data. Another approach in modeling data with a high zero frequency would be through the use of a zero-inflated distribution. In a mixture setting, zero-inflated models correspond to fitting one more component with an appropriate parameter. The distribution of this component can be considered of a Poisson form with parameter $\lambda=0$. Recently, zero-inflated models have become quite popular (see, e.g. Lambert, 1992; Li et al., 1999; Böhning et al., 1999 and the references therein).

\section{DISCUSSION}

The moment method is a widely used method mainly because of its simplicity. As was demonstrated in Section 3, the method is not efficient in the case of 2-finite Poisson mixtures. Moreover, in practice, it often fails to provide parameter estimates. These two issues make the method less attractive for practical purposes in comparison to the ML method, which always leads to parameter estimates and is computationally feasible via the EM algorithm. 
In this paper remedies for the cases where the moment estimates are not obtainable such as fixing one of the parameters to a specific value were not discussed, as the properties of such estimates are not known.

Replacing the third moment equation by the zero frequency equation, the estimation can be improved, mainly in efficiency, when the sample mean is small. This fact makes the method of zero frequency an interesting alternative to the ML for small values of the mean.

In this paper only the case of 2-finite Poisson mixtures was treated. The results for the general case of a $k$-finite Poisson mixture are no better, mainly because of the use of higher order sample moments. Looking at Table IIIC one can see that for a 3-finite Poisson mixture moment estimates are not obtainable for small to moderate sample sizes in nearly $80 \%$ of the cases.

In conclusion, the method of moments seems not to be a suitable choice of method for estimation in finite Poisson mixtures.

\section{Acknowledgement}

The authors would like to thank the Associate Editor and a referee for their comments on an earlier version of the paper.

\section{References}

Blischke, W. R. (1964). Moment estimators for the parameters of a mixture of two binomial distributions. Annals of Mathematical Statistics, 33, 444-454.

Blischke, W. R. (1965). Estimating the parameters mixtures of the binomial distribution. Journal of the American Statistical Association, 59, 510-528.

Böhning, D. (1999). Computer Assisted Analysis of Mixtures \& Applications in Meta-Analysis, Disease Mapping \& Others, C R C Press, New York.

Böhning, D., Dietz, E., Schlattmann, P., Mendonca, L. and Kirchner, U. (1999). The zero-inflated Poisson model and the decayed, missing and filled teeth index in dental epidemiology. Journal of the Royal Statistical Society, A 162, 195-209.

Brockett, P. (1977). Approximating moment sequences to obtain consistent estimates of distribution functions, Sankhya, A 39, 32-44.

Cohen, A. C. (1967). Estimation in mixtures of two normal distributions. Technometrics, 9, 15-28.

Day, N. E. (1969). Estimating the components of a mixture of normal distributions. Biometrika, 56, 463-474.

Douglas, J. B. (1980). Analysis with standard contagious distributions. Statistical Distributions in Scientific Work Series, Vol. 4. International Cooperative Publishing House, Fairland, Maryland, USA.

Everitt, B. S. and Hand, D. J (1981). Finite Mixtures Distributions, Chapman and Hall, New York.

Gupta, S. and Huang, W. T. (1981). On mixtures of distributions: A survey and some new results on ranking and selection. Sankhya, B 43, 245-290.

Harris, I. (1991). The estimated frequency of zero for a mixed Poisson distribution. Statistics and Probability Letters, 12, 371-372.

Hasselblad, V. (1969). Estimation of finite mixtures from the exponential family. Journal of the American Statistical Association, 64, 1459-1471.

Heckman, J., Robb, R. and Walker, J. (1990). Testing the mixture of exponentials hypothesis and estimating the mixing distribution by the method of moments. Journal of the American Statistical Association, 85, 582-589.

Heckman, J. (1990). A nonparametric method of moments estimator for the mixture of exponentials model and the mixture of geometrics model. In: Barnett, W. A., Powell, J. and Tanchen, G. (Eds.), Nonparametric and Semiparametric Estimation Models in Econometrics and Statistics. Cambridge University Press, pp 243-258.

Heckman, J. and Walker, J. (1990). Estimating fecundability data on waiting times to first conception. Journal of the American Statistical Association, 85, 283-294.

John, S. (1970). On analysing mixed samples. Journal of the American Statistical Association, 65, 755-760.

Johnson, N. L., Kotz, S. and Kemp, A. W. (1992). Univariate Discrete Distributions, 2nd ed., Wiley

Kano, Y. (1999). Delta method approach in a certain irregular condition. Communications in Statistics-Theory and Methods, 28, 789-807.

Karlis, D. (1998). Estimation and hypothesis testing problems for finite Poisson mixtures. Unpublished PhD Thesis, Department of Statistics, Athens University of Economics, ISBN: 960-7929-19-5.

Kemp, C. D. and Kemp, A. W. (1988). Rapid estimation for discrete distributions. Statistician, 37, $243-255$.

Lambert, D. (1992). Zero-inflated Poisson regression, with an application to defects in manufacturing. Technometrics, 34, 1-14. 
Li, C. S., Lu, J. C, Park, J., Kim, K., Brinkley, P. A. and Peterson, J. P. (1999). Multivariate zero-inflated Poisson models and their applications. Technometrics, 41, 29-39.

Lindsay, B. (1989). Moment matrices: Application in mixtures. Annals of Statistics, 17, 722-740.

Lindsay, B. and Basak, P. (1993). Multivariate normal mixtures: A fast consistent method of moments. Journal of the American Statistical Association, 88, 468-475.

Lindsay, B. and Roeder, K. (1992). Residuals diagnostics for mixture models. Journal of the American Statistical Association, 87, 785-794.

Panaretos, J. (1981). Unique properties of some distributions and their applications. Statistica, XLI, 567-574.

Pearson, K. (1894). Contributions to the mathematical theory of evolution. Philosophical Transactions of the Royal Society of London, 185, 71-110.

Quandt, R. and Ramsey, J. (1978). Estimating mixtures of normal distributions and switching regressions (with discussion). Journal of the American Statistical Association, 73, 730-752.

Rider, P. (1961). The method of moments applied to a mixture of two exponential distributions. Annals of Mathematical Statistics, 32, 143-147.

Rider, P. (1962). Estimating the parameters of mixed Poisson, binomial and Weibull distributions by the method of moments. Bulletin of the International Statistical Institute, 39, 225-232.

Shohat, J. and Tamarkin, J. (1943). The problem of moments. American Mathematical Society, New York.

Sichel, H. S. (1951). The estimation of the parameters of a negative binomial distribution with special reference to psychological data. Psychometrika, 16, 107-127.

Stuart, A. and Ord, K. (1994). Kendall's Advanced Theory of Statistics, 6th ed., Volume I., Wiley.

Tallis, G. M. and Light, R. (1968). The use of factorial moments for estimating the parameters of a mixed exponential distribution. Technometrics, 10, 161-175.

Tan, W. Y. and Chang, W. C. (1972). Some comparisons of the method of moments and the method of maximum likelihood in estimating parameters of a mixture of two normals. Journal of the American Statistical Associations, 62, 702-708.

Titterington, D. M, Smith, A. F. M. and Makov, U. E. (1985). Statistical Analysis of Finite Mixtures Distributions, Wiley.

Tucker, H. G. (1963). An estimate of the compounding distribution of a compound Poisson distribution. Theory of Probability and Applications, 8, 195-200.

Withers, C. S. (1991). Moment estimates for mixtures with common scale. Communications in Statistics- Theory and Methods, 20, 1445-1461.

Withers, C. S. (1996). Moment estimates for mixtures of several distributions with different means or scales. Communications in Statistics- Theory and Methods, 25, 1799-1824.

\section{APPENDIX: DERIVATION OF ASYMPTOTIC VARIANCE COVARIANCE MATRICES FOR THE PARAMETER ESTIMATES OBTAINED IN SECTIONS 2, 3 AND 5}

\section{A.1 The Maximum Likelihood Method}

For a 2-finite Poisson distribution the variance covariance matrix of the ML estimates can be found in the usual way via the information matrix. The vector $\boldsymbol{\theta}$ of parameters is $\boldsymbol{\theta}=\left(p_{1}, \lambda_{1}, \lambda_{2}\right)$. It is well known that the variance-covariance matrix for the ML estimates is the inverse of the information matrix, $\mathbf{I}(\boldsymbol{\theta})$ whose elements are:

$$
I_{i j}=-n E\left(\frac{\partial^{2} \ln (f)}{\partial \theta_{i} \partial \theta_{j}}\right)=-n E\left(H_{i j}\right)
$$

For the case of 2-finite Poisson mixtures the elements $H_{i j}$ are given by the following formulas:

$$
\begin{aligned}
& H_{11}=\frac{-\left(f_{1}(x)-f_{2}(x)\right)^{2}}{g^{2}(x)} \\
& H_{12}=H_{21}=\frac{\left(f_{1}(x-1)-f_{1}(x)\right) g(x)-p_{1}\left(f_{1}(x-1)-f_{1}(x)\right)\left(f_{1}(x)-f_{2}(x)\right)}{g^{2}(x)} \\
& H_{13}=H_{31}=\frac{\left(f_{2}(x-1)-f_{2}(x)\right) g(x)-p_{2}\left(f_{2}(x-1)-f_{2}(x)\right)\left(f_{1}(x)-f_{2}(x)\right)}{g^{2}(x)}
\end{aligned}
$$




$$
\begin{aligned}
& H_{22}=\frac{p_{1}\left(f_{1}(x-2)-2 f_{1}(x-1)+f_{1}(x)\right) g(x)-p_{1}^{2}\left(f_{1}(x-1)-f_{1}(x)\right)^{2}}{g^{2}(x)} \\
& H_{33}=\frac{p_{2}\left(f_{2}(x-2)-2 f_{2}(x-1)+f_{2}(x)\right) g(x)-p_{2}^{2}\left(f_{2}(x-1)-f_{2}(x)\right)^{2}}{g^{2}(x)} \\
& H_{23}=H_{32}=\frac{-p_{1} p_{2}\left(f_{1}(x-1)-f_{1}(x)\right)\left(f_{2}(x-1)-f_{2}(x)\right)}{g^{2}(x)}
\end{aligned}
$$

where $f_{i}(x)=f\left(x \mid \lambda_{i}\right)=\exp \left(-\lambda_{i}\right) \lambda_{i}^{x} / x !, i=1,2, g(x)=p_{1} f_{1}(x)+p_{2} f_{2}(x)$, and $p_{2}=\left(1-p_{1}\right)$

Then the elements of the information matrix are calculated as

$$
I_{i j}=n \sum_{x} H_{i j} g(x)
$$

The inverse of the matrix $\mathbf{I}$ is the variance-covariance matrix for the ML estimates.

\section{A.2 The Moment Method}

The asymptotic variance-covariance matrix of the estimators can be calculated as follows. Using a first order Taylor expansion (see, e.g., Titterington et al., 1985), the asymptotic variance covariance matrix $\mathbf{V}$ can be calculated as $\mathbf{V}=\mathbf{G}^{-1} \mathbf{M G} \mathbf{G}^{-1}$, where

$$
\mathbf{G}=\left[\begin{array}{ccc}
\lambda_{1}-\lambda_{2} & p_{1} & p_{2} \\
\lambda_{1}^{2}+\lambda_{1}-\left(\lambda_{2}^{2}+\lambda_{2}\right) & p_{1}\left(2 \lambda_{1}+1\right) & p_{2}\left(2 \lambda_{2}+1\right) \\
\lambda_{1}^{3}+3 \lambda_{1}^{2}+\lambda_{1}-\left(\lambda_{2}^{3}+3 \lambda_{2}^{2}+\lambda_{2}\right) & p_{1}\left(3 \lambda_{1}^{2}+6 \lambda_{1}+1\right) & p_{2}\left(3 \lambda_{2}^{2}+6 \lambda_{2}+1\right)
\end{array}\right]
$$

and $\mathbf{M}$ is the $3 \times 3$ matrix with its $i j$ th element representing the covariance between the $i$ th and $j$ th sample moments. Following Stuart and Ord (1994), the elements $M_{i j}$ of $\mathbf{M}$ are

$$
M_{i j}=\frac{1}{n}\left(E\left(X^{i+j}\right)-E\left(X^{i}\right) E\left(X^{j}\right)\right) .
$$

For a 2-finite Poisson mixture the first six simple moments are given by the following formulas

$$
\begin{aligned}
E(X) & =p_{1} \lambda_{1}+p_{2} \lambda_{2} \\
E\left(X^{2}\right) & =p_{1}\left(\lambda_{1}^{2}+\lambda_{1}\right)+p_{2}\left(\lambda_{2}^{2}+\lambda_{2}\right) \\
E\left(X^{3}\right) & =p_{1}\left(\lambda_{1}^{3}+3 \lambda_{1}^{2}+\lambda_{1}\right)+p_{2}\left(\lambda_{2}^{3}+3 \lambda_{2}^{2}+\lambda_{2}\right) \\
E\left(X^{4}\right) & =p_{1}\left(\lambda_{1}^{4}+6 \lambda_{1}^{3}+7 \lambda_{1}^{2}+\lambda_{1}\right)+p_{2}\left(\lambda_{2}^{4}+6 \lambda_{2}^{3}+7 \lambda_{2}^{2}+\lambda_{2}\right) \\
E\left(X^{5}\right) & =p_{1}\left(\lambda_{1}^{5}+10 \lambda_{1}^{4}+25 \lambda_{1}^{3}+15 \lambda_{1}^{2}+\lambda_{1}\right)+p_{2}\left(\lambda_{2}^{5}+10 \lambda_{2}^{5}+25 \lambda_{2}^{3}+15 \lambda_{2}^{2}+\lambda_{2}\right) \\
E\left(X^{6}\right) & =p_{1}\left(\lambda_{1}^{6}+15 \lambda_{1}^{5}+65 \lambda_{1}^{4}+90 \lambda_{1}^{3}+31 \lambda_{1}^{2}+\lambda_{1}\right) \\
& \quad+p_{2}\left(\lambda_{2}^{6}+15 \lambda_{2}^{5}+65 \lambda_{2}^{4}+90 \lambda_{2}^{3}+31 \lambda_{2}^{2}+\lambda_{2}\right) .
\end{aligned}
$$

In order to calculate the asymptotic efficiency of the $\mathrm{MM}$, the variance-covariance matrix of the ML method is required. 


\section{A.3 The Zero Frequency Method}

The asymptotic variance covariance matrix of the estimators using a first order Taylor approximation is calculated as $\mathbf{V}=\mathbf{G}^{-1} \mathbf{M} \mathbf{G}^{-1}$, where

$$
\mathbf{G}=\left[\begin{array}{ccc}
\lambda_{1}-\lambda_{2} & p_{1} & p_{2} \\
\lambda_{1}^{2}+\lambda_{1}-\left(\lambda_{2}^{2}+\lambda_{2}\right) & p_{1}\left(2 \lambda_{1}+1\right) & p_{2}\left(2 \lambda_{2}+1\right) \\
\exp \left(-\lambda_{1}\right)-\exp \left(-\lambda_{2}\right) & -p_{1} \exp \left(-\lambda_{1}\right) & -p_{2} \exp \left(-\lambda_{2}\right)
\end{array}\right]
$$

and $\mathbf{M}$ is the matrix with elements $M_{i j}$ given by $M_{i j}=(1 / n)\left(E\left(X^{i+j}\right)-E\left(X^{i}\right) E\left(x^{j}\right)\right)$. For $i, j=1,2$ these are the covariances of the sample moments (see Stuart and Ord, 1994). Also,

$$
\begin{aligned}
& M_{33}=\frac{1}{n} g(0)(1-g(0)) \\
& M_{13}=M_{31}=-\frac{1}{n} g(0)\left[p_{1} \lambda_{1}+\left(1-p_{1}\right) \lambda_{2}\right] \\
& M_{23}=M_{32}=-\frac{1}{n} g(0)\left[p_{1}\left(\lambda_{1}^{2}+\lambda_{1}\right)+\left(1-p_{1}\right)\left(\lambda_{2}^{2}+\lambda_{2}\right)\right] .
\end{aligned}
$$

where $g(0)=p_{1} \exp \left(-\lambda_{1}\right)+\left(1-p_{1}\right) \exp \left(-\lambda_{2}\right)$, i.e. $g(0)$ is the probability of 0 for a 2 -finite Poisson mixture. The above formulae can be derived using the appropriate formulae for the covariances of sample proportions given in Stuart and Ord (1994). 